\title{
Cambio climático y vulnerabilidad: prospectivas para la región nororiental de Colombia - Santanderes ${ }^{1}$
}

\author{
Jorge Enrique Pinto Hernández ${ }^{2}$ \\ Melva Salazar de Cardona ${ }^{3}$
}

\begin{abstract}
Resumen
Según las Naciones Unidas, el cambio climático supone efectos adversos, referidos a cambios en el medio ambiente físico o en la biota, con consecuencias nocivas significativas en la composición, la capacidad de recuperación y la productividad de los ecosistemas naturales o sujetos a ordenación, así como en el funcionamiento de los sistemas socioeconómicos, o en la salud y el bienestar humano. Se analizan aquí, cuáles son los tipos de vulnerabilidad que se observan en la población de los Santanderes.
\end{abstract}

Palabras clave: cambio climático, desarrollo sostenible, Santanderes, vulnerabilidad.

\footnotetext{
1. El presente estudio hace parte del macroproyecto denominado «Estado y prospectiva de las posibilidades de adaptación y mitigación de impacto del cambio climático en diferentes regiones del país», realizado por el Centro de Investigación en Medio Ambiente y Desarrollo y la Maestría en Desarrollo Sostenible y Medio Ambiente de la Universidad de Manizales.

2. Decana Escuela de Ciencias Humanas Artes y Humanidades. Universidad Nacional Abierta y a Distancia UNAD. Correo: sandra.morales@unad.edu.co

3. Docente Asistente de tiempo completo. Escuela de Ciencias de la Educación. Líder grupo de investigación UBUNTU. Universidad Nacional Abierta y a Distancia UNAD.

Correo: pablo.munevar@unad.edu.co
} 


\title{
Climate change and vulnerability: prospects for northeastern Colombia - Santanderes
}

\begin{abstract}
According to the United Nations, the climate change supposes adverse effects referred to changes in the physical environment or in the biota with harmful significant consequences in the composition, the capacity of recovery or the productivity of the ecosystems natural or subject to arrangement, or in the functioning of the socioeconomic systems, or in the health and the human well-being. It is studied here, which are the types of vulnerability that is observed in the population of the Santanderes.
\end{abstract}

Keywords: Climate change, sustainable development, Santanderes, vulnerability.

Recibido: 08/08/2014

Aceptado: 06/11/2014

\section{Introducción}

El aumento constante en el promedio mundial de la temperatura del planeta, registrada desde 1850, muestra mediciones que señalan el año 2010 como el año más caluroso junto con 1998 y 2005. Más allá de estos datos, la gran mayoría de la gente en el mundo, reconoce que el cambio climático, provocado por este calentamiento global, es un desafío muy serio para la humanidad y un tema de suma importancia para todos los países y sus gobiernos. Independiente de cuál sea su origen: si es natural o antropogénico, es importante establecer la vulnerabilidad de los pueblos y la capacidad de adaptación para superar estos cambios del clima, así como el establecimiento de acciones para mitigar los impactos generados por los efectos adversos del clima como son los desastres naturales, entre otros, según lo estableció la organización de las Naciones Unidas, en su convención anual de 1992 y que dio a conocer al mundo.

Para entender el fenómeno de cambio climático, es importante partir de la definición dada por la Convención de las Naciones Unidas sobre el mismo como «un cambio en el clima, atribuible directa o indirectamente a la actividad humana, que altera la composición de la atmósfera mundial y que se suma a la variabilidad climática natural observada durante períodos de tiempo comparables» (Naciones Unidas, 1992). Este cambio supone efectos adversos, referidos a cambios en el medio ambiente físico o en la biota con consecuencias 
nocivas significativas en la composición, la capacidad de recuperación o la productividad de los ecosistemas naturales o sujetos a ordenación, o en el funcionamiento de los sistemas socioeconómicos, o en la salud y el bienestar humano (Naciones Unidas, 1992). Frente a los efectos adversos debemos prepararnos para disminuir o mitigar el impacto del calentamiento global, realizar los análisis de vulnerabilidad para la toma de acciones y fortalecer la capacidad para ajustarse al cambio climático en lo que se denomina adaptación.

Los retos que derivan del cambio climático han motivado esta investigación, que tuvo como objetivos analizar e interpretar las acciones que se vienen realizando en la región de los Santanderes para disminuir los efectos adversos del cambio climático y la capacidad de superación de las regiones para afrontar las situaciones derivadas de dicho cambio, con el fin de reducir la vulnerabilidad de la población. Por lo anterior se plantearon los siguientes interrogantes: ¿Cuáles son las acciones que se vienen realizando en los departamentos de Santander y Norte de Santander para disminuir la vulnerabilidad a los efectos adversos del cambio climático? y ¿Cuáles son los tipos de vulnerabilidad que se observan en las poblaciones de los Santanderes? (Universidad de Manizales, 2010).

Para dar solución a estos interrogantes, se propusieron los siguientes objetivos: Describir las acciones de reducción de la vulnerabilidad al cambio climático, desarrolladas a nivel público y privado, en las áreas de la región nororiental del país, y tipificar las diferentes vulnerabilidades al cambio climático que se observan en las distintas poblaciones de la región de los Santanderes. El progreso de esta investigación sobre cambio climático, impulsó y promovió el desarrollo de la cooperación regional, para la investigación interdisciplinaria, sobre aquellos aspectos que relacionan directamente el cambio climático con las ciencias sociales, esto permitió conocer las posibilidades de reducir la vulnerabilidad de las diferentes poblaciones, principalmente, las ubicadas en la región de los Santanderes, El presente artículo está orientado al análisis de la vulnerabilidad y su tipificación, con una mirada holística e interdisciplinaria, que se constituye en un verdadero aporte a la comprensión de la problemática de la región.

\section{Marco referencial}

Por otra parte, el problema que se ha venido planteando, que es de características globales, se describe ampliamente en el Informe sobre Cambio Climático (IPCC, por sus siglas en inglés), correspondiente al año 2007, el cual fue realizado por la Comisión para el cambio climático de las Naciones Unidas. Este informe contiene los originadores de los procesos climáticos, detalla bajo una perspectiva de probabilidades las causas y efectos de dichos procesos, así como los factores de los cuales dependen y de manera probabilística también, las posibilidades de adaptación y mitigación. 
También, es importante mencionar, como lo señala el Instituto de Hidrología, Meteorología y Estudios ambientales IDEAM, que el cambio climático se debe, en parte, al incremento de las emisiones de Gases Efecto Invernadero GEI; aclarando que existe una diferencia entre variabilidad climática y cambio climático. La primera aparece cuando, con cierta frecuencia, un fenómeno genera un comportamiento anormal del clima, pero es un fenómeno temporal y transitorio; mientras que el cambio climático, se asocia a un proceso que no es temporal y que puede verificarse en el tiempo revisando datos climáticos, señalan que IPCC, describe un enfoque integrado del cambio climático teniendo en cuenta la dinámica del ciclo completo de causas y efectos interrelacionados en todos los sectores afectados (IDEAM, 2008).

Los expertos plantean en el informe mencionado, muchas de las probables consecuencias que conlleva el cambio climático: «El aumento del nivel del mar traerá como consecuencia daños a la infraestructura y salinización de cuerpos de agua dulce como ciénagas, estuarios y manglares. Las lluvias aumentaran su frecuencia y su magnitud debido al aumento en la rata de evaporación en los cuerpos de aguas superficiales, así mismo este aumento de la evaporación del agua traerá como resultado el aumento en la intensidad de las tormentas y huracanes. La agricultura se verá afectada a causa de la reducción de la humedad de los suelos por el alto índice de evaporación poniendo en riesgo la seguridad alimentaria. Las zonas tropicales se extenderán hacia latitudes más altas trayendo como consecuencia el desplazamiento de insectos y otras plagas transmisoras de enfermedades como el dengue, la malaria y la fiebre amarilla, aumentando el número de muertes por dichas enfermedades. La intensidad de la precipitación de las lluvias arrastrará material de los terrenos por escorrentía y se aumentará la sedimentación de sólidos en los cuerpos de agua, trayendo como consecuencia inundaciones y que muchos ríos de flujo permanente podrían secarse en algunas épocas del año con los consecuentes daños en la flora y la fauna. La pérdida de humedad de los suelos por efecto de la evaporación podría generar áreas desérticas y escases de agua que podría convertir los terrenos, en no aptos para la agricultura y la ganadería» (IPCC, 2007).

En el estudio del cambio climático también deben considerarse las consecuencias en el desarrollo humano, como lo enfatiza el informe sobre Desarrollo Humano 2007; «todo desarrollo busca ampliar las potencialidades humanas y aumentar las libertades; también busca desarrollar las capacidades que les permitan tomar decisiones y llevar una vida que consideren valiosa. En este sentido, el cambio climático amenaza con erosionar las libertades humanas y reducir las opciones, al mismo tiempo que pone en tela de juicio ese principio básico que sostiene que "gracias al progreso humano el futuro siempre será mejor que el pasado"»" (PNUD, 2007).

En Colombia y según el documento CONPES 3242 sobre cambio climático, el énfasis debe ponerse en los aspectos de adaptación y superación de la 
vulnerabilidad, según se desprende del Anexo A sobre «Cambio Climático», el cual se refiere así a la Convención sobre Cambio Climático: «Con el fin de enfrentar el cambio climático, la comunidad internacional adoptó el 9 de mayo de 1992, la Convención Marco de las Naciones Unidas para el Cambio Climático, que entró en vigor en 1994 y hasta la fecha ha sido ratificada por 188 países, entre ellos Colombia. Su objetivo es la estabilización de las concentraciones de gases de efecto invernadero en la atmósfera a un nivel que impida interferencias antropógenas peligrosas en el sistema climático» (DNP, 2003).

En este marco de referencia, la vulnerabilidad se define como: «la predisposición o susceptibilidad física, económica, política o social que tiene una comunidad de ser afectada y de sufrir daños en caso de que se manifieste un fenómeno desestabilizador de origen natural o antropogénico» (Cardona, 2001), y para Alberto «La vulnerabilidad es la capacidad intrínseca de cualquier componente del espacio geográfico, a ser afectado por una amenaza, como los desastres naturales, aunque el deterioro no ocurrirá si no existe un grado de vulnerabilidad. Esta depende del grado de exposición, de la protección o factores de prevención, de la reacción inmediata, de la recuperación básica del mínimo vital y de la reconstrucción de las partes implicadas en el suceso adverso que produjo el desequilibro ambiental» (Alberto, 2009).

Así interpretada, «la vulnerabilidad en sí misma forma un sistema dinámico y, por consiguiente, la interacción de las distintas variables implicadas determina la imposibilidad de los componentes, de un espacio geográfico dado para responder y reaccionar ante la presencia de un riego determinado, facilitando su destrucción» (Alberto, 2009). Es decir tiene que ver con situaciones que se dan antes, durante y consecutivamente al hecho catastrófico; por lo tanto repercuten circunstancias previas que, por ejemplo, «en el caso de comunidades vegetales y animales tienen que ver con sus amplitudes ecológicas para soportar cambios en sus condiciones ambientales (temperatura, humedad, deshidratación, etc.); mientras dura el suceso, donde influyen el tiempo y la dimensión del sometimiento al desastre que los afecta, y después del hecho destructivo; donde inciden factores como la capacidad de regeneración en lo natural o, de rápida solución y reorganización en lo social. Así el vocablo "vulnerabilidad, expresa tanto la exposición del riesgo como la magnitud de la capacidad, de cada elemento, para enfrentarlo a través de una respuesta"» (Alberto, 2009).

Comprender la vulnerabilidad al cambio climático requirió elaborar un marco conceptual básico sobre la temática, y ubicarlo en el ámbito de las regiones y los actores sociales implicados. Para ello, en este referente teórico, se siguió lo planteado por Wilches-Chaux (1989). Desde su teoría, «La Vulnerabilidad se debe considerar desde tres perspectivas o especificidades: vulnerabilidad natural, ecológica y física», no obstante corresponde tener presentes las otras que componen la tipología indicada por este autor es decir «vulnerabilidad social, económica, política, cultural, educativa e ideológica», ya que todas estas hacen de la vulnerabilidad, una situación global (Wilches, 1989). 
«La vulnerabilidad natural de los ecosistemas implica aptitudes y limitaciones de sus componentes, tanto bióticos como abióticos, vinculadas al acerbo genético de los organismos (plantas, animales y microbios) que hacen adaptaciones morfológicas, fisiológicas y conductuales, como también a las características fisiográficas del medio en el cual están insertos y con el cual interactúan. Esta vulnerabilidad se acrecentó sustancialmente con el incremento en el número de seres humanos, con su propia expansión sobre la tierra y sus avances en lo tecnológico y cultural para apropiarse de espacio, pues incitó a la resistencia humana a circunstancias ambientales severas, haciéndola muchas veces más vulnerable frente a ellas, dado que la población humana necesita de ciertas condiciones para poder desarrollarse, las que en la mayoría de los casos atentan contra el equilibrio dinámico, de la naturaleza» (Alberto, 2009). Enfoque este, que orienta a los ecologistas.

Parafraseando a Alberto, el surgimiento del concepto de la vulnerabilidad ambiental (ecológica), está vinculado estrechamente con la forma de convivencia de los grupos humanos y el ambiente, de tal forma que se geste una vulnerabilidad de los ecosistemas, frente a los efectos directos o indirectos de la acción humana y a los riesgos provocados por las comunidades que los explotan o habitan. En complementación a este concepto, surge la idea de vulnerabilidad física, referida a la localización de grupos humanos en áreas de riesgo, motivadas por la pobreza y falta de oportunidades para conseguir una ubicación mejor, lo que determina la sujeción y exposición de las sociedades a condiciones ambientales desfavorables por la localización de asentamientos en zonas expuestas a sucesos naturales cíclicos, ciertamente previsibles pero inevitables (inundaciones pluviales y fluviales, aludes, corrimientos de suelos, incendios, sismos, etc.), estos hechos van ligados a las características sociales e ideológicas de cada comunidad, a políticas permisivas y a una falta de educación e información respecto al funcionamiento de los sistemas ecológicos y de la naturaleza. Todo esto con un atenuante grave que los va a potencializar que es el cambio climático derivado del calentamiento global (Alberto, 2009), enfoque que orienta a los ambientalistas.

Una consecuencia del cambio climático es la alta vulnerabilidad de las poblaciones y su poca capacidad adaptativa a los cambios; esta circunstancia está directamente relacionada con el desarrollo social y económico de las sociedades, lo que implica que las más pobres y menos desarrolladas deberán sufrir de una forma más inclemente las consecuencias del cambio climático, con incidencia en aspectos ambientales como la seguridad alimentaria, el acceso al agua potable y el incremento a la morbilidad (IPCC, 2007).

\section{Metodología utilizada}

Se trató de una investigación de enfoque mixto: empírico-analítica, pues pretendió analizar, describir y tipificar, el fenómeno de la vulnerabilidad y hermenéutica, 
en tanto pretendió comprender las posibilidades de diminución del grado de vulnerabilidad en las poblaciones desde su propio contexto.

La unidad de análisis estuvo comprendida por las acciones que se realizan para disminuir el grado de vulnerabilidad, al cambio climático, y las tipificaciones de estas vulnerabilidades en las diferentes comunidades que hacen parte de la región nororiental del país. La unidad de trabajo estuvo compuesta por dos departamentos de la región nororiental del país, con características bien diferenciadas en las cuales se recogió la información necesaria para su caracterización. Este contexto, al que se suscribe el objeto de estudio, fue seleccionado de acuerdo a la ubicación de los estudiantes de la Maestría en Desarrollo Sostenible y Medio Ambiente, de la Universidad de Manizales, que decidieron participar en el estudio, y que hacen parte del macro proyecto de investigación titulado «Estado y prospectiva de las posibilidades de adaptación y mitigación de impacto del cambio climático en diferentes regiones del país».

\section{Fases de la investigación:}

Fase empírico -analítica:

Comprendió la recolección y análisis de información primaria y secundaria. Inicialmente en una caracterización de la región, que permitió tipificar las vulnerabilidades al cambio climático, y realizar el análisis de acciones emprendidas en la región nororiental del país, para tratar de disminuir dicha vulnerabilidad. Para este fin se plantearon hipótesis construidas a partir del referente teórico, las cuales fueron verificadas o falseadas en la medición de las diferentes variables atinentes a los comportamientos, actitudes, acciones y condiciones socio económicas de las poblaciones de los departamentos de Santander y Norte de Santander. Igualmente se incluyeron los sistemas de información geográfica SIG para, sistematizar e intercambiar información, datos, redes existentes y cruzarla con la información cualitativa, esto sirvió para ubicar prácticas y acciones emprendidas en el territorio en torno a la vulnerabilidad al cambio climático.

Fase descriptiva:

Asociada a la anterior, en esta fase se procedió a la caracterización de las vulnerabilidades al cambio climático contempladas desde lo público y privado, a partir de la identificación de patrones, y de modelos que permitieron reflejar los comportamientos y la tipificación de los tipos de vulnerabilidad que se generan en la región nororiental del país.

\section{Fase hermenéutica:}

En esta fase se determinó, con base en las fases anteriores cuantitativas, las categorías cualitativas que permitieron interpretar las tipificaciones de vulnerabilidad de las diferentes comunidades al cambio climático, y las condiciones en que se dan las vulnerabilidades en las poblaciones de los 
Santanderes. Esta fase se realizó mediante diálogos direccionados a través de entrevista con informantes claves que conocen de la problemática en la región, con grupos focales y con comunidades vulnerables de Girón, Santander. Igualmente se tuvieron en cuenta las autoridades implicadas en el manejo del medio ambiente, tales como las corporaciones autónomas regionales, los jefes de planeación, las organizaciones de base constituidas para la atención de poblaciones vulnerables ante el cambio climático.

Se aplicaron encuestas a los funcionarios responsables de los temas ambientales, y/o de cambio climático, de las diferentes alcaldías que hacen parte de la región de los Santanderes, con el fin de dar respuesta a los interrogantes que permitieran conocer las actividades que se realizan en sus dependencias, y que tienen articulación con las políticas relacionadas con el cambio climático establecidas en el POT y/o plan de desarrollo local. Para esto fueron diligenciadas 110 encuestas en línea, teniendo en cuenta el cargo que ocupa cada funcionario que participó, de lo que se encontró que el $23,6 \%$ de los encuestados correspondió a cargos altos; el 37,2\% cargos medios y el $39.09 \%$ a cargos bajos. En cuanto a las dependencias que participaron en la encuesta en línea, se encontró en un 48,2\% dependencias altas, 39,09\% dependencias medias y un $11,8 \%$ dependencias bajas ${ }^{4}$.

\section{Técnicas de recolección de información:}

En las fases empírico - analítica y descriptiva, se procedió a la revisión documental en las corporaciones autónomas regionales y demás entidades para determinar qué empresas están trabajando en la temática y cuáles acciones se están desarrollando en los niveles estatal y privado. Además implicó la elaboración y aplicación de técnicas de recolección de información, como: encuestas dirigidas a la comunidad, para tipificar la vulnerabilidad de las poblaciones. Para la recolección de la información se recurrió a fuentes primarias y secundarias, considerando información documental del Ministerio de Medio Ambiente y Desarrollo Sostenible MADS, del Instituto de Hidrología, Meteorología y Estudios Ambientales IDEAM, de las corporaciones autónomas regionales y entidades del orden regional, planes de desarrollo departamentales y municipales, entrevistas y participación en foros, entre otras.

En la fase hermenéutica se procedió a la aplicación de entrevistas con preguntas abiertas para actores claves y la realización de grupos focales con comunidades vulnerables y con representantes de las instituciones. Se aplicaron encuestas a los funcionarios responsables de los temas ambientales y/o de cambio climático

\footnotetext{
4. Cargos altos: alcaldes, gobernadores, concejales, diputados, directores de corporaciones, secretarios de despacho, directores de UMATAS, Directores grupos de investigación, directores de nodos regionales, etc. Cargos medios: asistentes de secretarías, investigadores, subdirectores de corporación y, nodos regionales, consultores, profesionales universitarios, asistentes, etc. Cargos bajos: auxiliares, secretarios, técnicos de UMATAS, practicantes, etc.

Dependencia alta: alcaldías, gobernaciones, concejos, asambleas, secretarías, planeación, UMATA, Federación Nacional de Cafeteros, desarrollo económico, desarrollo territorial, Dependencia media: subdirecciones, asistencia técnica, obras públicas, asistencia técnica UMATA, gestión de riesgo y desastres, despachos, unidad de desarrollo rural, Dependencia baja: comunera, rural, minería, protección social, técnicos, practicantes (Encuesta).
} 
de las diferentes alcaldías que hacen parte de la región de los Santanderes. De los 87 municipios del departamento de Santander respondieron 71 funcionarios en 48 municipios, es decir en el 55\%, mientras que en el departamento de Norte de Santander, compuesto por 40 municipios, respondieron 39 funcionarios en 33 municipios, es decir en el $82.5 \%$. El foro se utilizó para validar la información, acción adelantada el 21 de febrero de 2013, con participación de Corponor, Alcaldía de Ocaña por Norte de Santander y Corambiente por Santander.

\section{Análisis y discusión de resultados}

\section{Prospectivas para la superación de la vulnerabilidad}

En Santander se identifican dos grandes unidades fisiográficas: la del Valle del Magdalena, con un modelado plano y suavemente ondulado y la Cordillera Oriental, que ocupa casi todo el departamento, la cual se caracteriza por un relieve quebrado y de pendientes fuertes; en el flanco occidental de la cordillera sobresalen terrazas de ambientes secos (Otero, et al., 2012). Igualmente la región de Norte de Santander tiene una geografía variada, compuesta por serranías, páramos, mesetas, llanuras y cerros, con municipios de diferentes alturas; esto la hace inmensamente rica en paisajes y climas. A lo largo de su territorio la recorren ríos y lagunas. En el departamento se caracterizan tres regiones diferentes: la primera, la Serranía de los Motilones, caracterizada por ser una región muy quebrada en la que hay partes altas, aún cubiertas con selva; la segunda está compuesta por el ramal que se desprende del nudo de Santurbán, presentando alturas de hasta $3.329 \mathrm{~m}$, como el páramo de Tamá; y la tercera, la correspondiente a la vertiente y valle del Catatumbo, es una región muy húmeda, de altas precipitaciones y con su población bastante dispersa (Parques Nacionales de Colombia, 2005).

En Santander el clima es variable, debido a la topografía y la humedad que predominan en las diferentes subregiones, con pisos térmicos que van desde el cálido con alturas hasta el páramo alto,presente en dos ecosistemas, el valle del Magdalena Medio y la zona Andina, correspondiente a la cordillera oriental del sistema de los Andes. Se diferencian entre sus ecosistemas: páramos, serranías, valles, selva andina, bosque alto andino, bosques secos, humedales ciénagas, lagunas, ecosistemas secos, xerofíticos, selvas hidrofíticas, entre otros. Se identifican 27 ecosistemas estratégicos, distribuidos en 1.141.687 ha (Gobernación de Santander 2008-2011, 2011). Por su parte en Norte de Santander, se destacan ecosistemas estratégicos como el Parque Regional Natural Sisavita, Complejo Lagunar Santurbán y el Páramo de Almorzadero, de los cuales sólo el parque Sisavita tiene un área aproximada de 12.000 has, con el 14\% de la biodiversidad de los Andes, lo que habla de la importancia en términos de biodiversidad y agua para Norte de Santander (Corponor, 2010). 
El Plan Nacional de Cambio Climático, presentado en agosto de 2012 por el Ministerio del Ambiente y desarrollo sostenible (MADS), el cual entre otros, recomienda que sea incorporado en los sectores y los entes territoriales «el cambio climático» dentro de sus políticas, planes, programas y proyectos (MADS, 2012). En los planes de desarrollo de la región, se han incorporado aspectos sobre el mismo: en el caso de Santander (2012-2015), se proponen unas líneas de acción en torno a la adaptación al cambio climático. En el plan de desarrollo de Norte de Santander se registra un eje para el desarrollo territorial, «un Norte Sostenible» el cual esboza algunos proyectos que abordan el calentamiento global y promueven una alianza para la consolidación del nodo de adaptación al cambio climático (Gobernación de Santander, 2012).

La actual administración departamental de Santander prevé avanzar en un 30\% el proceso de implementación y operación del Sistema de Información Geográfica SIG departamental y la meta al cuatrienio la proponen en un $60 \%$ (Gobernación de Santander, 2012). Por otra parte la CDMB cuenta con un Sistema de Información Ambiental SIA, en el cual han adoptado un Sistema de Información Geográfico; dentro de los aplicativos de SIA, se publican mapas de biodiversidad para las cuencas de la jurisdicción de la CDMB e información técnica y espacial de la Red de Monitoreo de la Calidad del Agua (Corporación Autónoma Regional para la Defensa de la Meseta de Bucaramanga, 2012). Como complemento de los sistemas de información, la página de la Corporación enlaza al Sistema de Información Ambiental de Colombia SIAC (Sistema de Información Ambiental de Colombia, 2012). Por su parte Norte de Santander ha iniciado el proceso de actualización del Sistema de Información Geográfica (SIG) de acuerdo a resolución 000046 de Mayo 08 de 2012, que lo coloca en plan de actualización permanente de las herramientas que le permiten la actualización de los análisis y modelamientos espaciales, especialmente en temas de administración de los recursos naturales y de medio ambiente.

Respecto a la condición de gestión del riesgo para la región Santanderes, se cuenta con la Oficina de Prevención y Atención de Desastres de la CAS; en general las autoridades ambientales solicitan a los diferentes municipios contar con planes municipales de gestión de riesgo activos, anteriormente llamados planes de atención y prevención. La Corporación Autónoma Regional para la Defensa de la Meseta de Bucaramanga CDMB cuenta con el programa «reducción de riesgo y su gestión» (CDMB, 2011), y en Norte de Santander, Corponor ha ofrecido asesoría y acompañamiento a las oficinas de gestión de riesgo de cada municipio, así como el asesoramiento a los diferentes alcaldes y delegados municipales para abordar la estructuración y formulación de los instrumentos de planificación en el marco de la normatividad, haciendo énfasis en la incorporación de la Gestión del Riesgo de Desastres con base en la Ley 1523 de 2012. 
Adicionalmente se encuentran los nodos regionales para el cambio climático para la región de los Santanderes, el cual hace parte del Nodo Norandino y lo conforman, la CAS, CDMB y Corponor (González Lamus, Pabón, \& Duarte Ortega, 2011), (López y Toro, 2011); estos nodos han sido impulsados nuevamente por el Ministerio de Medio Ambiente y Desarrollo Sostenible y son concebidos como «grupos regionales interinstitucionales e interdisciplinarios de trabajo, conformados por personal de instituciones públicas y privadas del orden local, departamental y regional, que trabajan articuladamente bajo la coordinación del ministerio, buscando contribuir a la formulación, promoción, articulación y desarrollo de políticas, lineamientos y estrategias interinstitucionales, en materia de cambio climático» (Ministerio de Medio Ambiente y Desarrollo Sostenible, 2012); el Nodo Norandino, que fue constituido en el año 2010 cuando se adelantó el primer taller en el marco del cual se constituyó y se elaboró el plan de acción del mismo (octubre 2010) (Nodo Regional de Cambio Climático Norandino, 2010), ha logrado avanzar en estos dos últimos años mediante la realización de talleres y encuentros; se destaca la reunión adelantada a finales de 2012, convocada por el Ministerio de Medio Ambiente conjuntamente con la WWF Colombia, el cual centró la agenda en «Analizar y definir una estructura institucional - arreglos entre las entidades- que facilite la gestión de medidas de mitigación y adaptación al Cambio climático»; de igual forma busca integrar el Nodo regional de cambio climático Norandino, y plantear mecanismos operativos para su funcionamiento; avanzar en la consolidación del Plan de Acción del Nodo regional de cambio climático Norandino (Tomado de la agenda taller «Análisis y fortalecimiento de estructura institucional y del plan de acción del nodo regional de cambio climático Norandino»; San Juan de Girón; diciembre 5 y 6 de 2012).

A partir de esta caracterización y su comparación con los resultados obtenidos, mediante todas las fuentes de información utilizadas, se puede decir que los datos son confiables y permiten trabajar el tema de la vulnerabilidad, analizando las políticas, la articulación de temas relacionados con el cambio climático, la coherencia de las políticas de Estado con las políticas de los gobiernos departamentales y locales, y la evaluación de actividades dirigidas a reducir las vulnerabilidades en las comunidades. La encuesta arrojó que las actividades realizadas en estas dependencias tienen cierta articulación con las políticas relacionadas con el cambio climático establecidas en el Plan de Ordenamiento Territorial POT y/o Plan de Desarrollo Local. Existe cierta coherencia entre el conocimiento de la vulnerabilidad de la región y la actuación política de los dirigentes de la zona, es decir, el tema del cambio climático está presente en las agendas gubernamentales, pero no de una manera explícita, como lo corroboran los grupos focales institucionales indagados.

Las actividades que se realizan en las dependencias encuestadas, tienen articulación con las políticas relacionadas con el cambio climático establecidas 
en el POT y/o Plan de desarrollo, y en su mayoría son la reforestación, el manejo de licencias de impacto ambiental, educación y concientización, reducción de emisiones de gases de efecto invernadero, uso racional del agua, obras de infraestructura, programas de prevención y atención a los desastres, plan de desarrollo, plan de acción, estudio de amenazas y riesgo, implementación de tecnologías con sostenibilidad ambiental, y control de recursos hídricos.

En el caso del Nodo Norandino conformado por las corporaciones autónomas Corporación Autónoma Regional de Santander, Corporación Autónoma Regional para la Defensa de la Meseta de Bucaramanga y Corponor (Corporación Autónoma regional de Norte de Santander), se realizan actividades formuladas dentro del Plan de Desarrollo de Santander 2012-2015, que propone unas líneas de acción en torno a la adaptación al cambio climático. Se tiene por ejemplo, el caso del municipio de Girón, que desde el año 2010 se encuentra un estudio de actualización, contratado por la CDMB y una empresa privada, sobre la amenaza por inundación del río de Oro, sector Bahondo hasta la confluencia con el río Suratá. Se puede decir que hay un conocimiento de las políticas o directrices orientadas a disminuir la vulnerabilidad al cambio climático en la región y en su mayoría se identifican con la adaptación a la variabilidad del cambio climático: plan de gestión de riesgos, campañas encaminadas a la educación ambiental, POMCH, POTS, PGAR, Plan de Acción, Plan Nacional de Desarrollo, Plan Departamental de Desarrollo, Plan de Desarrollo Municipal.

En los Santanderes se han fortalecido las oficinas de gestión de riesgo, que es la instancia encargada de brindar en los municipios la respuesta a eventos relacionados con la prevención y atención de desastres. Se observa un mayor interés por parte de las autoridades locales en esta oficina, y para desarrollar las actitudes positivas frente al cambio climático de la población de Norte de Santander, se crearon, los comités técnicos institucionales; el comité técnico interinstitucional de Educación Ambiental, promovido por el Ministerio de Educación Nacional y el Ministerio de Ambiente y Desarrollo Sostenible para la implementación de la Política Nacional de Educación Ambiental; el CIDEA, ha formulado varios planes de educación ambiental. Los aspectos de mitigación al cambio climático que se están trabajando en la región son la formulación, gestión y desarrollo de proyectos en mitigación, reforestación, identificación y buen uso de las zonas de protección.

El comité se constituye en el espacio interinstitucional de la educación ambiental y es este punto el que mejor permite conocer la realidad de cada localidad y región. Al observar la dinámica de estos espacios de discusión y debate, se puede decir que existe cierta conciencia en los funcionarios encargados de los temas ambientales entre las instituciones sobre la vulnerabilidad de la región hacia el cambio climático, pero ellos no creen que las instituciones y mucho menos las poblaciones del área de su influencia, se encuentren preparados para adaptarse a este tipo de fenómenos derivados del cambio climático y superarlos de manera 
adecuada, así lo dejaron ver, en su participación a través de los grupos focales de estudio y la contrastación con el trabajo de los comités técnicos institucionales ${ }^{5}$.

Ahora bien, la coordinación interinstitucional y el trabajo interdisciplinario para reducir la vulnerabilidad al cambio climático, solo puede entenderse bajo la mirada de la complejidad del contexto en el que se desarrolla y esta mirada la ofrece, de manera clara, la caracterización de esta región. La región Nororiental del país o los Santanderes la componen 127 municipios, de los cuales 87 pertenecen a Santander, en seis provincias (Vélez, Comunera, Guanentina, García Rovira, Soto y Mares); mediante la Ordenanza 00304 del 6 de diciembre de 2005, se constituyeron ocho Núcleos de Desarrollo Provincial (Metropolitano, Carare Opón, Soto Norte, Comunera, Guanentina, García Rovira, Mares, Vélez), los cuales buscan ser instrumento de gestión para la promoción del desarrollo económico y social integral. Mientras que el departamento de Norte de Santander fue creado como tal, mediante la ley 14 del 25 de julio de 1910 y comprendía las provincias de Cúcuta, Ocaña, Pamplona y 25 municipios. Actualmente cuenta con 40 municipios que conforman su base política administrativa y la unidad territorial objeto del plan de gestión territorial (ESAP, 2004-2007). Esta región es altamente vulnerable, respecto al cambio climático, en cada uno de los aspectos tipificados por Wilches Chaux (1989) y otros, como las pérdidas de cultivos y cosechas, desplazamiento en sitios donde es imposible el agua, y riesgo de deslizamientos. También se conocen experiencias significativas que evidencian acciones tomadas en las localidades para adaptarse a los efectos del cambio climático en energía alternativa, creación de sistemas y procesos de reforestación.

Este inventario que se tiene de la oferta institucional, pública y privada, para atender el tema ambiental y lo relacionado con el fenómeno del cambio climático, permite la construcción de prospectivas para la superación de la vulnerabilidad, el análisis de esta oferta institucional, permite observar la alta disposición en la región de personal suficientemente calificado para realizar estas acciones de reducción a la vulnerabilidad de las poblaciones y superar los daños de los impactos nocivos actuales y futuros. Esta prospectiva la dejan ver los funcionarios a cargo y está sistematizada en los apartes del trabajo con los grupos focales institucionales: "Los funcionarios conocen bien los planes de contingencia en su localidad para enfrentar los efectos adversos del cambio climático, las obras y acciones de protección para los ciudadanos, planes de precaución, y el plan de gestión de riesgo» ${ }^{6}$.

Visto de esta manera, por ejemplo, en el caso de Santander, las posibilidades de superar la vulnerabilidad en la población depende del cumplimiento de las metas

5. Los comités participan de la realización de proyectos para cultura ambiental, obras y acciones de protección para la población, campañas de educación ambiental y reforestación, mejoramiento de vivienda, protección de las fuentes hídricas campañas para incentivar a las comunidades, uso eficiente del agua, y proyectos de mitigación (Encuesta).

6. El clima ha modificado las costumbres de la comunidad en los últimos años, se perciben temperaturas más altas, cambio en la forma de vestir por el cambio climático, nuevas formas de aprender a reciclar y proteger las fuentes de agua, movilidad y desplazamiento, incertidumbre en las cosechas y cambios en la forma de cultivar (Encuesta). 
establecidas a 2015 por el departamento de Santander en el Plan de Desarrollo denominado Santander en serio, el gobierno de la gente 2012-2015, del cual, vale la pena resaltar: el proceso de formulación de los planes de ordenación y manejo de dos cuencas hidrográficas, la ejecución de tres planes de manejo de cuencas hidrográficas ordenadas, la declaratoria de 60.000 hectáreas de áreas protegidas en el departamento de Santander, la reforestación y aislamiento de 1.500 hectáreas de las áreas de protección de micro cuencas abastecedoras de acueductos, la conservación ecológica de tres cañadas en Bucaramanga, el desarrollo de seis proyectos relacionados con producción sostenible (sistemas agroforestales y silvopastoriles) y producción limpia, el desarrollo de cuatro proyectos que incrementan la oferta de bienes y servicios ambientales (agroturismo), la gestión para la consolidación en un $100 \%$ del sistema departamental de áreas protegidas, la implementación de 500 hectáreas de bosques endoenergéticos, el establecimiento de 3.000 hectáreas de bosque comercial, la suscripción de dos convenios con las universidades regionales para que se adelanten procesos que garanticen la política pública del medio ambiente.

\section{La vulnerabilidad al cambio climático en las poblaciones de los Santanderes}

Los resultados del estudio permiten analizar el grado de conocimiento que se tiene en la población de la variación del clima, los pobladores son conscientes que el clima ha venido cambiando últimamente pero no se desarrolla aún en ellos una conciencia histórica, por ejemplo: en el departamento de Santander el $63,4 \%$ dice conocer las políticas orientadas a disminuir la vulnerabilidad al cambio climático en su región, sin embargo este tema lo confunden con temas ecológicos o ambientales, mientras que en el departamento del Norte de Santander el conocimiento fue del $41 \%$. Estas políticas están fundamentadas en las actividades de adaptación a la variabilidad del cambio climático, plan de gestión de riesgos, campañas encaminadas a la educación ambiental, Plan de Acción, Plan Nacional de Desarrollo, Plan Departamental de Desarrollo y Plan de Desarrollo Municipal.

La caracterización socioeconómica y ambiental de la región permitió, entre otros aspectos, conocer los planes de desarrollo de los departamentos que consolidan las acciones y metas planificadas en la gestión ambiental y del riesgo, que de una u otra manera apuntan a la mitigación de los efectos adversos del cambio climático, a mejorar la capacidad de adaptación de la región para afrontar las relaciones derivadas de dicho cambio, con el fin de reducir la vulnerabilidad de la población, como se mencionó anteriormente. Los estudios que respaldan los planes de desarrollo contienen datos del nivel de vulnerabilidad de las poblaciones, se puede extraer de ellos, frente a qué fenómenos se presenta la vulnerabilidad y a quienes afecta en menor o mayor grado y así lo confirman los resultados de la encuesta, se tiene claro el concepto de vulnerabilidad en la población 
y si esta tiene o no relación con el fenómeno del cambio climático; para la variable inundaciones, el 52,1\% y 48,7\% respondieron Alta vulnerabilidad para Santander y Norte de Santander respectivamente. Para la variable deslizamiento respondieron Alta vulnerabilidad en un 77,5\% y 64,1\% para Santander y Norte de Santander respectivamente; mientras que para la variable sequias la encuesta arrojo en un $42,3 \%$ y $59 \%$ como Alta vulnerabilidad para Santander y Norte de Santander. Se puede decir que el nivel de vulnerabilidad de la región frente a los impactos del cambio climático es alto, según la percepción de los encuestados, (gráficos 1 y 2).

\section{Alta vulnerabilidad - Santander}

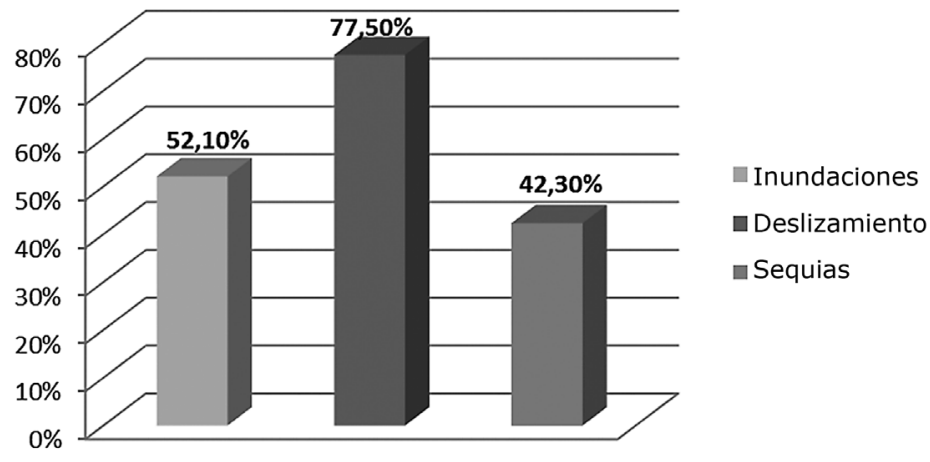

Gráfico1. Resultados de las encuestas / Santander.

La gran mayoría de los funcionarios entrevistados, alrededor del $80 \%$, consideraron que las actividades que se realizan en su dependencia, están articuladas con las políticas relacionadas con el cambio climático, establecidas en el POT y/o Plan de Desarrollo Local, en aspectos tales como la reforestación, manejo de licencia de impacto ambiental, educación y concientización, reducción de emisiones de gases de efecto invernadero, uso racional del agua, obras de infraestructura, programas de prevención y atención a desastres, plan de desarrollo, plan de acción, estudio de amenazas y riegos, implementación de tecnologías con sostenibilidad ambiental y control de recursos hídricos. Frente a la vulnerabilidad en su región, respecto al cambio climático en cuanto a los aspectos de inundación, deslizamientos, sequías y afectación a la vivienda, consideran en un alto porcentaje que son altamente vulnerables, mientras que en los aspectos de disminución de glaciares e incremento del nivel del mar, la gran mayoría considera que representan una baja vulnerabilidad. Así mismo, en los aspectos pérdida de especies de flora y fauna, pérdida de empleo, seguridad alimentaria y afectación a centros educativos, los clasifican entre mediana y alta vulnerabilidad. 


\section{Alta vulnerabilidad - Norte de Santander}



Esta vulnerabilidad al cambio climático determinada para la región, que es alta, aparece definida respecto a cada uno de los aspectos o tipificaciones de la vulnerabilidad que se da en cada localidad o municipalidad de acuerdo a la clasificación que propone Wilches Chaux en su trabajo. El nivel de vulnerabilidad aparece de manera general y esto obedece, según se pudo constatar en las entrevistas realizadas a los funcionarios encargados de las acciones ambientales, al grado de descoordinación que hay en torno al tema del cambio climático y sus efectos. Por ejemplo: se confunden los aspectos ecológicos con los aspectos ambientales, y por lo general se le atribuyen a los efectos del cambio climático, todos los aspectos relacionados con desastres naturales como; inundaciones, deslizamientos, sequias, disminución de glaciares, incremento del nivel del mar, pérdida de especies de flora y fauna, pérdida de empleo, afectación a la vivienda, problemas de salud, seguridad alimentaria, afectación a centros educativos, entre otros. Esta manera de pensar, o la ausencia de una base del pensamiento ambiental en la cultura de la región, se manifiesta en la poca interdisciplinariedad a la hora de intervenir en un proceso educativo ambiental, que ligue las acciones con los problemas relacionados y las vulnerabilidades a los efectos del cambio climático, y es por esto que aparecen acciones ecológicas y ambientales diseminadas por los planes de desarrollo locales y regionales, sin un eje temático que los articule, ni un marco conceptual que oriente las posibilidades de superación de esas vulnerabilidades, como lo propone Wilches Chaux (1989).

Entonces, se tiene que los impactos que ha generado el cambio climático en la región, de acuerdo con la tipificación propuesta por Wilches Chaux, se pueden resumir en un aumento de la temperatura, cambios en la producción agrícola, fuerte olas de calor, variabilidad del clima, sequía o inundaciones según el caso, inestabilidad económica, infraestructura, deterioro progresivo de los recursos 
naturales, deslizamiento, desplazados, escasez de agua. La vulnerabilidad económica se manifiesta en los daños en cultivos, gastos inesperados, cambios de cultivos, pérdidas de cultivos, muerte de especies, destrucción de vías, pérdida de vivienda y suelos productivos, disminución en los ingresos, gastos en infraestructura, migración a otros municipios (resultados de las encuestas).

La vulnerabilidad social se da en la pérdida de vivienda, calidad de vida, desempleo, desplazamiento y damnificados, problemas de salud, posibilidades de violencia y delincuencia. La vulnerabilidad ambiental se ha manifestado con los incendios forestales, erosiones, deslizamientos e inundaciones, efectos de deforestación por cambio de cultivos, marcadas temporadas de lluvia y sequías prolongadas, afectación y deterioro de bosques por incendios forestales, contaminación, pérdida de terrenos, cultivos, enfermedades, deterioro de fuentes hídricas.

Ahora bien, la vulnerabilidad política se refleja en el modo de vida, gestión de recursos, políticas públicas de la gestión del riesgo, desgaste político, falta de cultura ambiental de los dirigentes, incredulidad en las políticas nacionales y locales, concientización de la problemática, inestabilidad en el gobierno, mejoras de la normatividad, modificación en la inversión de recursos, desarrollo urbanístico, más atención a las políticas de protección al medio ambiente, falta presencia del Estado.

La vulnerabilidad cultural se ha dado en la modificación de prácticas culturales, los hábitos y la forma de vestir, rechazo a la adopción de nuevas tecnologías, cambios por la influencia del turismo, desarraigo, nuevas formas de vivir, conciencia ambiental. Otras vulnerabilidades se dan por la existencia de otros problemas sociales y pocos recursos para atenderlos, como en la salud, y la dificultad de movilización.

Así mismo, el 60\% de los encuestados conoce los planes de contingencia en su localidad para enfrentar los efectos adversos del cambio climático, manifestando verlos reflejados en las obras de acciones y protección para los ciudadanos, planes de precaución y plan de gestión del riesgo. Sin embargo en la región de los Santanderes, el 70\% afirma que los lineamientos políticos de los últimos gobernantes en su localidad no han aportado a que las entidades y la comunidad en general tome medidas tendientes a cambiar hábitos y estilos de vida para minimizar los efectos del cambio climático, ya que las políticas no son aplicadas y es necesario un gran trabajo de socialización y concientización para lograr propósitos institucionales, no hay visión de protección ambiental, planes para mitigar los efectos del cambio climático, cultura ambiental, jornadas de reforestación o protección de los recursos naturales (resultados de las encuestas).

Las entrevistas y las reuniones con grupos focales permitieron conocer bajo estos mismos lineamientos el panorama sobre cambio climático de quienes trabajan 
en el tema, así como quienes han vivido sus efectos como en el caso de los habitantes de la ribera del Río de Oro en el municipio de Girón que vivieron la avalancha del año 2005. Se pudo establecer con estas entrevistas, la preocupación por los impactos que genera el cambio climático en la región y el alto nivel de vulnerabilidad a la que está expuesta la población. Uno de los damnificados, hablando de su percepción frente a los planes y acciones gubernamentales dice «considero que obedecen a acciones aisladas y débiles, que no dan respuesta la magnitud de los riesgos» (Informante 1) es decir, presentan un panorama de corrección y no de prevención. Se calcula que las acciones de prevención tienen un costo muy bajo en comparación a los costos para corregir los daños una vez se presenta una catástrofe, esto sin contar que nunca se recupera en su totalidad, sin embargo nuestros gobiernos prefieren abordar los problema una vez que se presentan, quizá probablemente porque las obras de prevención no generan tantos votos como el asistencialismo.

El análisis de la oferta institucional, en comparación con la percepción que tiene la gente de las acciones que se hacen en torno al tema de la ecología o al tema ambiental, demuestra que existe información clara y disponible sobre estos efectos del cambio climático y los tipos de vulnerabilidad que se presentan en cada una de las poblaciones, se dan tal como lo propone Wilches Chaux en su propuesta teórica sobre la vulnerabilidad.

\section{Superación de la vulnerabilidad.}

La tarea de determinar cuáles son los sectores económicos y sociales más vulnerables de la región nororiental y por qué se consideran más vulnerables, tratados en el aparte anterior, contrastando los resultados de la encuesta con el marco teórico que propone Wilches Chaux, ha dependido del concepto de cambio climático y la percepción de vulnerabilidad de las poblaciones, este concepto varía de acuerdo a los intereses de cada uno de los actores sociales y su contexto, estas visiones o formas de ver el mundo van a determinar las acciones que se realizan en Santander y Norte de Santander, y que permiten enfrentar los efectos adversos del cambio climático y determinar la eficacia y eficiencia de la inversión pública y privada cómo la capacidad de adaptación de esta región para afrontar la situación que se deriva de dicho cambio y lógicamente, es importante todo esto buscando reducir esa vulnerabilidad a que se enfrenta la población y que se hace susceptible a esos cambios.

Esta cosmovisión que se desarrolla en la vida cotidiana de nuestras gentes, es precisamente lo que propone Wilches Chaux y que permite tipificar los diferentes niveles de vulnerabilidad que se observan en las distintas regiones, permite conocer las condiciones mitigantes de los impactos del cambio, que pueden estar presentes en las poblaciones vulnerables y su disposición a la prevención, y comprender las posibilidades de adaptación y superación de las diferentes 
regiones al cambio climático, convirtiéndose así, este referente teórico, como la base de un pensamiento ambientalista para el trabajo interdisciplinario.

El marco referencial propuesto por Wilches Chaux, fue también la base para comprender la discusión en el foro realizado, de manera virtual, con profesionales de diferentes disciplinas y relacionados con el tema del cambio climático. Este espacio permitió validar la información recolectada y dejó ver la percepción que tienen los que se pudieran considerar «expertos en el tema», coincidente con la percepción que tiene el ciudadano, la gente de a pie, frente al cambio climático. Comenta uno de los participantes al foro, refiriéndose al cambio climático «pues es una palabra relativamente nueva, acuñada tan solo unos años atrás, y le estamos echando la culpa al cambio climático y por cuenta de nosotros mismos, de las actividades y las intervenciones del tiempo antrópico, por ejemplo; en los parques nacionales, donde se ha modificado el ambiente, donde el clima no será el mismo. A la gente le preocupan los cerros orientales de cada ciudad, saben que no se ha empezado hacer nada para proteger las reservas naturales de los invasores, que estamos invadiendo una zona o reserva natural porque es necesario y ya. Saben de la minería a gran escala, que van a talar por hectáreas y esto a las comunidades no le parece que sea negociable, a las comunidades no las pueden negociar, les ha tocado desarrollar una ciudad que ha crecido desordenadamente, por cuenta de la mismas alcaldías y los gobiernos locales, sus dirigentes son los que han cerrado los ojos ante cualquier injusticia, lógicamente el cambio climático es una materialización de un riesgo como el de talar los bosques, intervenir las fuentes de agua, las micro cuencas, es el resultado de la ausencia de una conciencia histórica» (Profesional de Parques Nacionales que vive en Bucaramanga). La afectación por los cambios de clima en la actividad económica, en los recursos hídricos y la seguridad alimentaria, dependen más de la percepción de la realidad de un profesional que de estudios serios que respalden nuestra actividad como funcionarios públicos.

Otro Profesional comenta «El empoderamiento de las comunidades se refiere a su capacidad para auto determinarse, de apropiarse de una ley que les permita trabajar en conocimiento del riesgo, que es lo que lógicamente quieren sus habitantes y tienen que conocer su pasado, saber lo que ocurrió en nuestra ciudad o comunidad en un periodo de 50 o 25 años, donde hablar con las personas sea la base de la concertación y los acuerdos y tener en cuenta los escenarios y los riesgos, donde se pueda decidir las empresas que intervienen directamente con el cambio climático y los desarrollos urbanos, los cuales hay que empezar a intervenirlos y a reglamentar las áreas que son de reserva» (Profesional CAS). El desconocimiento de las prioridades y los sectores vulnerables al cambio climático como el económico, el agrícola, el eco sistémico, los recursos hídricos, la seguridad alimentaria, los aspectos socio-culturales, no permite la coordinación interinstitucional por la dificultad de establecer acuerdos cuando hay tantos intereses. 
Un comentario más de otro profesional participante en el Foro, a manera de ilustración: "El Catatumbo medio es una zona cercana a Ocaña donde hay organizaciones sociales construyendo muchas cosas que parecen pequeñas pero que han logrado generar un impacto en las políticas públicas, alli se tienen muchas preocupaciones frente al cambio climático y todas las afectaciones referentes a estas comunidades y todo viene de las políticas del Estado implementadas en los Santanderes para concesionar el territorio para la minería como una política de Estado. Esta problemática de la minería ha colocado nuestros departamentos como zona roja, la movilidad social en defensa del páramo de Santurbán en contra del proyecto de la mega minería Angosturas de explotación a cielo abierto, sigue siendo una amenaza en su entorno, inferior al cambio climático» (profesional del nodo). Las instituciones orientan las ayudas de acuerdo al interés de cada comunidad y son direccionadas por los dirigentes de turno, esto afecta las fuentes de ingresos y de alguna manera, se tiene la percepción que los cambios del clima han afectado esos ingresos. Hay, en cierta medida, un desconocimiento por parte de los profesionales encargados de los temas ambientales de las acciones de los Departamentos encaminadas a reducir el riesgo y los daños originados por el cambio climático.

Los profesionales que se consideran expertos en el tema del cambio climático, reunidos en el foro virtual, realizaron su aporte a la superación de las vulnerabilidades, determinando las causas de la ausencia de una política pública en el tema ambiental para la región, veamos un enfoque Ambientalista: "Las comunidades y los ciudadanos arremeten contra la destrucción de zonas de páramo, no entienden cómo puede haber un Estado con una política de gestión para el cambio climático y con unas comunidades de campesinos teniendo pocas cosas, cuando ya hay una organización que los viene o los está perjudicando en su hábitat, aqui en Santander esta lo del páramo de Santurbán y en Norte de Santander está el caso del Robledo, en el parque nacional donde allá hay problemas serios y una amenaza en todo lo relacionado con nacimientos de agua. La gente tiene sus principales preocupaciones en esos megaproyectos de minería que van en contra de lo que a la prevención o preparación o a la mitigación de lo que es el cambio climático, tanto en las comunidades urbanas como rurales, y los megaproyectos de grandes plantaciones que acaban con toda la zona de bosques pretendiendo hacer unos cambios de bosques lo cual con eso se va perdiendo toda la biodiversidad que ya todos conocemos» (apartes del Foro).

El enfoque ecologista está contenido en el siguiente aporte: «El tema de los parques Nacionales en Santander y Norte de Santander, afectados por el cambio climático, está orientado a las áreas protegidas con el tema de vulnerabilidad, de la alteración del habitad, de las especies exóticas invasoras, sobrexplotación y contaminación. Todos son impactos sobre estos ecosistemas donde la conservación y gestión de las áreas protegidas es una prioridad a nivel nacional, y se requiere de una estructura integral donde se evalúen todos los aspectos y factores de afectación a los ecosistemas, para orientar las políticas, 
los lineamientos públicos en las regiones territoriales y las áreas protegidas; nuestra labor esta en tomar esas políticas, esas estrategias y esos lineamientos $y$ convertirlos en politicas públicas que afecten la realidad de cada área protegida. La región nororiental tiene desde selvas húmedas tropicales como en el caso del Catatumbo, tenemos andinos, sub andinos, paramos, sub paramos, etc.» (apartes del Foro).

En esto ha radicado la complejidad de este análisis, en que «se tiene una diversidad del ecosistema con una riqueza cultural dentro de esas áreas protegidas, tenemos comunidades campesinas, comunidades indígenas y esto genera otros desafios para la gestión de la conservación, en manos de quienes son reconocidos en tema ambiental pero también en la autoridad nacional y regional; referente a las comunidades indígenas, la gestión es completamente diferentes que frente a la adaptación de las diferentes comunidades, la gestión se entiende diferente en las regiones autónomas, en las alcaldías, por los CAPIS que son los comités ambientales municipales, pues a partir de estas diferencias que se generan, las acciones frente al cambio climático, generan expectativas diferentes en los ciudadanos, como desarrollar los planes ambientales con lo local y lo regional y como construimos un aspecto técnico conceptual para hacer el ejercicio con el tema del medio ambiente a nivel social» (conclusiones del Foro).

El trabajo con los grupos focales y el haber propiciado un espacio de encuentro con los expertos en el tema, en un foro virtual, permite rescatar algunos puntos en los cuales se pudiera decir que hay consenso «Los consejos ambientales son la instancia donde se toman muchas decisiones, y que pudiesen materializarse en los planes de desarrollo cómo conservación de áreas estratégicas para los departamentos, el saneamiento predial con la comunidad, planeamiento predial, sistemas o ecosistemas emergentes, áreas de pastoreo, cultivos ilícitos y áreas o ecosistemas en desastre. Podemos decir que estas comunidades, pueden desarrollar su capacidad de empoderamiento para proteger y cuidar estas áreas protegidas y gracias a ellas les podemos dar este nombre» (Foro de expertos).

"Uno de los protagonistas principales de estos consejos ambientales son las organizaciones que están hablando a nivel rural, las asociaciones de mujeres y otras asociaciones han sido protagonistas y hay muchos ejemplos de la historia reciente del Páramo de Santurbán, donde se vio cómo una política del estado regional y nacional pretendía concesionar un proyecto de explotación minera y fue la organización de la sociedad civil la que se opuso a eso y allí se evidenció el empoderamiento de las comunidades para defender un ecosistema estratégico para la conservación de un sistema estratégico» (apartes tomadas del Foro virtual). Por aquí es el camino para vencer la vulnerabilidad; queda entonces seguir trabajando en el conocimiento de resultados exitosos en la superación de la vulnerabilidad, la búsqueda de insumos estadísticos confiables y profundizar en investigaciones propias de localidades y grupos étnicos que permitan seguir validando el pensamiento de Wilches Chaux. 


\section{Conclusiones}

La preparación que se tiene a nivel del departamento y municipios en el manejo de la vulnerabilidad, se refleja en el conocimiento que tienen sus autoridades frente a los diferentes mecanismos, herramientas, instrumentos, conceptos, con los que cuentan para abordar el cambio climático, pero ni los departamentos ni los municipios cuentan con los recursos suficientes que permitan en un momento dado desarrollar los ajustes necesarios para disminuir el nivel de vulnerabilidad, y preparar sectores estratégicos como el tema del agua, de alimentos, para preparar y desarrollar dinámicas de ajuste a estos efectos del cambio climático. Entonces, por una parte, hay una conciencia en las autoridades, pero por otra parte, hay muy baja comprensión de estas implicaciones en las comunidades campesinas y comunidades productoras; existe una voluntad política para involucrar estos temas, que son absolutamente estratégicos, dentro de las prioridades del desarrollo sostenible de la región nororiental.

La dificultad más grande que se tiene en el tema del cambio climático, es la interdisciplinariedad, que compromete distintas especialidades, distintos saberes e intereses. Esta dificultad se refleja en la metodología para el manejo de la problemática, que ha dotado el tema del cambio climático en cada una de las organizaciones públicas y privadas, determinando qué percepción se tiene en cada una de las poblaciones, en las entidades públicas y privadas, en cada uno de los entornos donde intervienen el tema de cambio climático y como aún no se han articulado las instituciones para redactar el tema del cambio climático, específicamente en Santander. Entonces queda abierta la posibilidad de dar una gran discusión. Se requiere de una transdisciplinariedad que aborde estas complejidades, que hacen que el problema de cambio climático sea altamente relevante en esta región.

Pensar el tipo de desarrollo sostenible que se requiere para los Santanderes, por no decir Colombia, hace necesario, en ese sentido, el seguir trabajado con comunidades vulnerables un modelo de desarrollo sostenible, enmarcado dentro de esa relación con el ambiente, de manera que lo asuman como una mejor forma posible de vida y en ese sentido garantizar la protección de cuencas, la organización social, la siembra de comida, el rescate de la economía campesina y la eliminación de cultivos ilícitos en las zonas, el manejo de las problemáticas de conflicto armado, la lucha territorial de los grupos al margen de la ley. Es desde una política pública donde las comunidades empiezan a conocer si realmente es conveniente, o no, esta política para cumplimiento de toda la población y no solo de algunas instituciones del Estado. Si se construyen acuerdos, el tema del cambio climático nos concierne a todos, no es solamente un problema de las instituciones del Estado pero sí son ellas quienes deben liderar un proceso de sensibilización y acompañamiento a todas las comunidades para que puedan afrontarlo de una manera adecuada. 
La zona de los Santanderes es más vulnerable, específicamente por la topografía de la región; sin embargo, las decisiones políticas que se toman al respecto del cambio climático son de tipo reactivo, existe una visión a atender más los eventos catastróficos, pero aún no están claros los lineamientos para una respuesta integral desde lo que se ha observado. La educación es fundamental para todas las etapas de formación del cambio climático, empezando con los niños y siguiendo con las universidades, haciendo del tema del cambio climático, un tema obligado. Se dice del cambio climático que es un tema de moda y dentro de poco tiempo no se volverá a hablar de él, pero realmente los problemas generados por el cambio climático son de gran envergadura que no van a pasar rápidamente, más que ser un tema de moda es un tema vital para la población. Es importante socializar todos los resultados de la investigación con los nodos de trabajo, allí es donde se nota un esfuerzo coordinado institucionalmente, para que el tema del cambio climático esté presente en todas las agendas de las gobernaciones y los gabinetes municipales en pleno, para que se apropie un poco más el tema de la vulnerabilidad y la responsabilidad sea compartida por las entidades de las regiones.

La vulnerabilidad desarrollada por comunidades al cambio climático, ha implicado un proceso de ajuste permanente en respuesta a las nuevas y diferentes circunstancias ambientales que se han presentado en los Santanderes, hasta el paisaje ha cambiado, en definidas cuentas es la incapacidad de un sistema para ajustarse al cambio climático, es la impotencia de las comunidades para disminuir los daños potenciales que han sufrido y tomar ventaja para retomar sus vidas. Tal ha sido el caso de las familias que viven a orillas del rio Girón, en la cabecera municipal de este municipio.

Crear una nueva ciencia del desarrollo ambiental, será una tarea de todos los que quieran trabajar en el tema del cambio climático, es necesario desarrollar una conciencia histórica en las personas que les desarrolle su capacidad de empoderamiento colectivo de las comunidades, frente a los temas adversos del cambio climático; el efecto invernadero y el otro, capturar el $\mathrm{CO}_{2}$, eso aplica para todo el planeta y los temas referidos a los efectos adversos donde el hombre viene y modifica la naturaleza. Con esa modificación hay una afectación después una amenaza y una vulnerabilidad. La conciencia histórica permite conocer las características de las poblaciones, sus necesidades, sus instituciones, su capacidad de solidaridad, y de alguna manera de tener una perspectiva de su futuro. En este sentido se deben orientar futuras investigaciones, estudio de localidades y estudios etnográficos. 


\section{Referencias bibliográficas}

Alberto, J. 2009. Vulnerabilidad ecológica... Entre lo ambiental y lo social. El caso del área metropolitana del Gran Resistencia. En: A.M. Foschiatti (comp.). Aportes conceptuales y empíricos de la vulnerabilidad global. Pp. 117- 158. Resistencia: Universidad Nacional del Nordeste. Disponible en: http://hum.unne.edu.ar/ publicaciones/instGeo/digitales/vulnerabilidades/archivos/cap4.pdf

Asamblea Departamental de Norte de Santander. 2012. Ordenanza $N^{\circ} 006$ del 13 de Junio de 2012, Por medio del cual se adopta el Plan de Desarrollo, Un Norte Pa Lante, 2012-2015. Cúcuta.

Asamblea Departamental de Santander. 2012. Ordenanza $N^{\circ} 013$ del 23 de Abril de 2012, Por medio del cual se adopta el Plan de Desarrollo, Santander en Serio, el Gobierno de la Gente, 2012-2015. Bucaramanga.

Asohofrucol. 2006. (M. D. MADR, Productor, \& Equipo Técnico Plan Frutícola Nacional De Colombia - PFN) Recuperado el 20 de Mayo de 2012, Disponible en: http://www.google.com.co/url?sa=t\&rct=j\&q=el\%2odepartamento\%2onorte\%20 de $\% 20$ santander $\% 20$ se $\% 20$ encuentra $\% 20$ conformado $\% 20$ por $\% 20$ tres $\% 20$ grandes $\% 20$ cuencas $\% 2$ oque $\% 2$ oson $\% 3$ A $\% 2$ la\%2ogran $\% 2$ ocuenca $\% 20$ del $\% 20$ catatumbo $\% 2$ C 20 la\%20gran\%20cuenca\%20del\%2omagdalena\%20y\%20la\%20

Cardona, O.D. 2003. La necesidad de repensar de manera holística los conceptos de vulnerabilidad y riesgo. En: La Red. Red de estudios sociales en prevención de desastres en América Latina. Disponible en: http://www.desenredando.org/public/ articulos/2003/rmhcvr/rmhcvr_may-08-2003.pdf

CDMB. 2011. Corporacion Autónoma Regional para la Defensa de la Meseta de Bucaramanga. Disponible en: http://www.cdmb.gov.co/web/index.php/gestion-delterritorio/gestion-del-riesgo.html Consultado el 22 de octubre de 2011.

Congreso de Colombia. 2011. Senado de la República de Colombia. de Información legislativa www.secretariasenado.gov.co : Disponible en: http://www. secretariasenado.gov.co/senado/basedoc/ley/1994/ley_0164_1994.html Consultado el 28 de mayo de 2011.

Corponor. 2010. Corponor. Disponible en: http://www.secretariasenado.gov.co/ senado/basedoc/ley/1994/ley_0164_1994.html Consultado el 26 de mayo de 2012.

Departamento Nacional de Planeación (DNP). 2003. Estrategia Institucional para la venta de servicios ambientales de mitigación del cambio climático. Consejo Nacional de Política Económica y Social. Documento CONPES 3242, República de Colombia,. Bogotá, Agosto 25 de 2003. Disponible en: http://www.minminas.gov.co/minminas/ downloads/UserFiles/File/AMBIENTAL/PoliticaAmbiental/Conpes\%203242.pdf 
Gobernación Norte de Santander. 2012. Gobernación Norte de Santander. Disponible en: http://www.nortedesantander.gov.co/infgeneral.php Consultado el 24 de mayo de 2012.

Gobernación de Santander. 2012. Gobernación de Santander. (13 de Abril de 2012). Dsiponible en: http://www.santander.gov.co/gobernacion_mm/estatica/images/ destacados/pag/pdd/index.php?sec=1 Consultado el 25 de Mayo de 2012.

IDEAM. (s.f.). cambioclimatico.gov.co. Disponible en: http://www.cambioclimatico. gov.co/descargas? com $=c c \&$ name $=$ pubFile $6588 \&$ downloadname $=$ Nodos Regionales_Para_documento_de_Vulnerabilidad-IDEAM-2-08-11.doc Consultado el: 25 de mayo de 2012.

IPCC. 2007. Cambio climático 2007: Informe de síntesis. Contribución de los Grupos de trabajo I, II y III al Cuarto Informe de evaluación del Grupo Intergubernamental de Expertos sobre el Cambio Climático [Equipo de redacción principal: Pachauri, R.K. y Reisinger, A. (directores de la publicación)]. Ginebra: IPCC. Disponible en: http:// www.ipcc.ch/pdf/assessment-report/ar4/syr/ar4_syr_sp.pdf

IPCC. 2008. Cambio Climático 2007 Informe de Sintesis (Primera Impresión, 2008 ed.). (IPCC, \& O. M. OMM, Edits.) Ginebra, Suiza: IPPC; OMM - Organización Metereológica Mundial.

López, M.C. \& Toro, B.M. 2011. Instituto de Hidrología,Metereología y Estudios Ambientales: Centro de Documentación e Información Cientifico Técnica. IAvH, IDEAM, IIAP, INVEMAR, SINCHI (Septiembre de 2011). Disponible en: https://documentacion. ideam.gov.co/openbiblio/Bvirtual/022166/022166.htm. Consultado el 1 de Febrero de 2013.

Naciones Unidas. 1992. Convención Marco de las Naciones Unidas para el Cambio Climático. FCCC/INFORMAL/84*.

Nodo Regional de Cambio Climático Norandino. 2010. Gestión integral de riesgo y mitigación y adaptación cambio climático. Minambiente; Cormacarena; Asocars, Santander, Bucaramanga.

Otero, J. A., Uribe, J.C., Hormiga, C.M., Rodríguez, L.A. 2012. Diagnóstico de Salud de Santander. (C. M. Hormiga, Ed.) Revista del Observatorio de Salud Pública de Santander, 7(1): edición especial.

Parques nacionales de Colombia. 2005. Plan de manejo Parque Nacional Natural Catatumbo-Bari. Disponible en: http://www.parquesnacionales.gov.co/PNN/portel/ libreria/pdf/PlandeManejoPNNCatatumboBari.pdf Consultado el 24 de Marzo de 2013. 
Programa de la Naciones Unidas para el Desarrollo PNUD. 2007. Informe sobre desarrollo humano, la lucha contra el cambio climatico: solidaridad frente a un mundo dividido. Nueva York: Programa de la Naciones Unidas para el Desarrollo PNUD. Disponible en: http://www.acnur.org/biblioteca/pdf/7709.pdf?view=1

Universidad de Manizales. 2010. (Grupo de Investigación Medio ambiente y desarrollo sostenible); estado y prospectiva de las posibilidades de adaptación y mitigación de impacto del cambio climático en diferentes regiones del país. Manizales (Noviembre de 2010).

UT. 2009. Unimineriapasm Departamento de Santander - Contrato Upme No. 020410312-2008. Programa de Aprovechamiento de Minerales - Pasm en el Departamento de Santander. Municipios de Barrancabermeja, Betulia, Simacota, El Carmen, San Vicente de Chucurí, San Gil, Oiba, Cirití, El Cerrito, Landazuri y Bolivar. Consultoría, UT - UNIMINERIA, Bogotá.

Vásquez, G. 2009. Cátedra Agraria: Modificación del Bioclima debido al cambio climático global. Universidad Nacional, sede Medellín. Departamento de Ciencias Forestales. Medellín.

Wilches-Chaux, G. 1989. Herramientas para la crisis: desastres, ecologismo y formación profesional. Popayán: SENA. 